\title{
Un rappel d'un rendez-vous 1997-2005 de partenaires syndicaux et patronaux du Saguenay-Lac saint-Jean et de leur déclaration d'engagement
}

\author{
Pierre Deschênes ${ }^{1}$ \\ Université du Québec à Chicoutimi
}

Il nous apparaît opportun, dans ce texte, de rappeler le cheminement d'un "atelier de recherche du futur ». Ce fut une démarche prospective et mobilisatrice utilisée en 1998 par des dirigeants syndicaux et patronaux pour élaborer un scénario du futur visant à développer pour 2005 un avantage stratégique à la création d'entreprises, à l'investissement économique et au développement du plein emploi au SaguenayLac-Saint-Jean. La démarche misait sur la concertation syndicale-patronale dans le monde du travail.

La «Future Search Conference ${ }^{2}$, ou l'atelier de recherche du futur, constitue une approche importante de concertation et de mobilisation réunissant, autour d'un thème commun, les principaux partenaires internes et externes d'une organisation, d'une communauté, d'une ville, d'une région. Cette stratégie permet d'obtenir des changements rapides pour une collectivité. Les conditions de ces changement rapides sont de mobiliser les personnes qui représentent le système dans sa totalité dans un endroit déterminé ( « in the room ») et, dans un moment très bref (deux jours et demi à trois jours), de les inviter à inventer par un dialogue social entre elles, sans avoir recours à des experts externes, un scénario du futur qui sera le résultat d'un consensus. La règle du consensus demeure également dans la détermination des actions permettant la réalisation du scénario du futur.

Du 8 au 10 janvier 1998, 60 acteurs majeurs représentant les dirigeant patronaux, syndicaux, socioéconomiques et socio-communautaires se sont réunis à la Dam-en-Terre sur les rives glacées du Lac Saint-
Jean et, pendant deux jours et demi, ont engagé un dialogue social, sans avoir recours à des experts externes, pour élaborer par consensus un scénario du futur avec un horizon de $2005^{3}$. Nous vous invitons, en premier lieu, à suivre la mobilisation des partenaires du travail du Saguenay-Lac-Saint-Jean qui a conduit à cette session de prospection du futur. Puis nous vous présentons le scénario 2005 qui a fait l'objet d'un consensus. Finalement, la conclusion propose de mettre le cap sur 2025 et de continuer de construire une culture de la concertation dans le monde du travail.

\section{La mobilisation de partenaires du travail}

C'est dans un contexte d'une région à la recherche d'un avantage stratégique qu'est né, au début de 1996, le projet de créer un rendezvous de partenaires du travail du Saguenay-LacSaint-Jean. Un regroupement informel de personnes préoccupées de la qualité des relations du travail a décidé d'amorcer une réflexion commune sur une perception répandue dans le milieu des entreprises. La venue de nouveaux capitaux pour la création de nouvelles entreprises serait tributaire d'une vision négative des investisseurs concernant une main-d'œuvre régionale perçue comme étant très revendicatrice. De juin à septembre 1996, des rencontres de mobilisation de dirigeants syndicaux et patronaux ont alors permis de dégager un consensus pour créer un forum régional de concertation syndicale-patronale afin de démontrer que cette vision négative des investisseurs n'a pas de fondement solide. 


\section{La mobilisation pour identifier des problèmes touchant le monde du travail}

En octobre 1996, un premier groupe informel de travail, composé de représentants patronaux et syndicaux, a reçu le mandat de rechercher un projet commun susceptible de rejoindre les préoccupations des dirigeants syndicaux et patronaux du Saguenay-LacSaint-Jean. Une première action de ce groupe a été de demander à un économiste de la direction régionale de la Société québécoise de développement de la main-d'œuvre (SQDM) d'effectuer une recherche sur les conflits de travail, les pertes de temps de travail découlant de ces conflits et autres phénomènes pouvant infirmer ou confirmer la perception négative des investisseurs. Cette étude a démontré clairement que la région du Saguenay-Lac-Saint-Jean se comparait avantageusement aux autres régions du Québec en matière de relations du travail et que sa main-d'œuvre n'est pas plus revendicatrice qu'ailleurs au Québec. En 2004, cette constatation nous apparaît être la même.

Devant ces résultats, le groupe de travail a délaissé l'idée de construire un projet commun basé seulement sur cette perception négative des investisseurs. Il a agrandi son champ d'analyse en recherchant d'autres facteurs affectant le développement des entreprises au Saguenay-Lac-Saint-Jean. Cette analyse, réalisée d'une manière impressionniste, a permis de dégager ces constats. Il y a beaucoup d'énergie humaine investie pour faire fonctionner et mettre en place des petites et moyennes organisations (PME). Les grandes entreprises manufacturières (Abitibi-Consolidated, Alcan) dont dépend la vie de la région sont en mutation constante. En ce qui concerne les organisations publiques et parapubliques, les hôpitaux sont rudement touchés par les compressions budgétaires et les départs massifs à la retraite. Le monde scolaire connaît le même phénomène doublé d'une baisse de clientèle. Le monde municipal est en effervescence avec les négociations entre le gouvernement et les municipalités.

En plus des syndiqués et des gestionnaires touchés par ces changements, le groupe de travail a surtout mis en évidence que les plus vulnérables demeurent, d'une part, les jeunes qui quittent la région devant la rareté des emplois, ce qui entraîne une perte d'un potentiel d'intelligence au service du développement de la région et, d'autre part, les exclus du monde du travail : les travailleurs en chômage, les assistés so- ciaux et autres. En 2004, ces constats demeurent d'une étonnante actualité.

\section{La région du Saguenay-Lac-Saint-Jean se compare avantageusement aux autres régions du Québec en matière de relations du travail.}

Sur la base de ces constats, des dirigeants patronaux et syndicaux ont décidé, au terme de plusieurs rencontres de mobilisation tenues de septembre 1996 à février 1997, qu'il était très opportun de mettre activement à contribution l'intelligence de femmes et d'hommes de cette région dans une démarche commune visant ces deux objectifs généraux :

- promouvoir, par des gestes concrets, les expériences de partenariat déjà existantes dans notre région afin de démontrer à l'ensemble des entreprises régionales et surtout à des investisseurs étrangers qu'au Saguenay-Lac-Saint-Jean, il se réalise des projets innovateurs dans les relations patronales-syndicales;

- parvenir éventuellement à une charte des relations du travail ou encore à un contrat social régional énumérant les grands principes ou les grands dénominateurs communs de l'engagement patronal-syndical; il s'agit en quelque sorte d'implanter progressivement une culture de concertation syndicale-patronale à la grandeur de la région du Saguenay-Lac-Saint-Jean.

$\mathrm{Au}$ cours d'une rencontre en février 1997, 56 leaders syndicaux et patronaux ont alors décidé par consensus d'utiliser une stratégie de prospection du futur afin de trouver des actions concrètes pour atteindre ces objectifs généraux

\section{La mobilisation pour préparer un atelier de recherche du futur}

De mars 1997 à janvier 1998, un comité paritaire patronal-syndical, nouvellement formé, a poursuivi le travail de mobilisation des partenaires du travail. Tout en maintenant le cap d'une consultation des partenaires du travail, le comité est parvenu à faire consensus sur quatre choix importants pour le déroulement de l'atelier de recherche du futur. Un premier consensus 
a été obtenu sur le thème de l'atelier : Le travail : un avantage stratégique à développer d'ici 2005 pour la région du Saguenay-Lac-Saint-Jean. Un deuxième consensus sur le but de l'atelier a été le suivant: D'ici 2005, les partenaires du travail du Saguenay-LacSaint-Jean devront développer, par des actions précises, un avantage stratégique régional basé sur les ressources humaines en misant sur la qualité des interactions et de la concertation dans le monde du travail.

Un troisième consensus a porté sur les quatre éléments retenus devant composer le scénario de ce qui est souhaitable pour la région du Saguenay-LacSaint-Jean d'ici l'an 2005: 1) la main-d'œuvre et l'emploi (ex. la formation, le reclassement, le développement de l'emploi), 2) l'organisation du travail (ex. la gestion des ressources humaines dans l'entreprise, le perfectionnement des travailleurs), 3) l'éthique et la valeur des partenaires par rapport au travail (ex. les conditions de travail, la qualité de vie au travail), et 4) les relations du travail. Finalement, un quatrième consensus a été dégagé quant au choix des actrices et des acteurs de l'atelier. Le choix de 64 personnes visait à regrouper les principaux leaders syndicaux, socio-économiques, communautaires ainsi que les propriétaires et les gestionnaires d'entreprises qui sont impliqués dans la grande, la moyenne et la petite entreprise des secteurs privé, public et parapublic.

\section{La mobilisation des forces vives de l'ensemble du territoire de la région a été un exercice difficile à réaliser.}

Nous voulions mobiliser simultanément des acteurs clés du monde du travail de la région du SaguenayLac-Saint-Jean. Il est moins complexe de réaliser une démarche de prospection du futur lorsque la frontière $\mathrm{du}$ système est bien circonscrite comme celle d'une organisation. La mobilisation des forces vives de l'ensemble du territoire de la région a été un exercice difficile à réaliser. Comment réunir dans un même endroit fermé, et pendant près de 24 heures, 64 personnes ayant des intérêts idéologiques, politiques, économiques communs, divergents et parfois conflictuels ? Bien que difficile, cet exercice a conduit au choix de personnes provenant majoritairement du monde patronal (24) et syndical (24) ainsi que du monde socio-communautaire (8) et du monde socioéconomique de la région et de l'extérieur de la région (8).

\section{Le scénario 2005 des partenaires du travail}

Devant le refus de partenaires importants de participer à l'atelier, le comité paritaire de coordination a hésité jusqu'à la dernière minute à tenir ou non l'atelier. Sans minimiser cette difficulté de parcours et en souhaitant créer un effet d'entraînement pour mobiliser après l'atelier ces partenaires récalcitrants, la session de prospection des partenaires du travail, un défi pour la région du Saguenay-Lac-St-Jean s'est déroulée pendant 16 heures les 8, 9 et 10 janvier 1998. Ces objectifs ont été poursuivis :

- dégager un consensus par rapport à un scénario ou une vision commune du futur pour la région de 1998 jusqu'en 2005;

- identifier les actions que les partenaires sont prêts à poser pour assurer la réalisation de cette vision.

$\mathrm{Au}$ terme de plusieurs exercices de prospection, les partenaires du travail sont parvenus à faire consensus sur ces quatre éléments d'un scénario du futur.

Le premier élément a trait aux valeurs et à l'éthique devant guider les actions des partenaires du monde du travail. Les partenaires ont alors convenu de définir, d'adhérer et de faire la promotion d'une vision globale du développement régional sans exclusion de personne ou de catégorie de personnes, basée sur une concertation régionale structurée autour des valeurs de respect mutuel, de transparence, de coopération et de partage entre les partenaires du monde de travail. Ce développement se fera en favorisant le plein emploi et le plein potentiel humain au travail. Il s'agit d'une quête d'un sens plus équitable et plus humain pour gérer notre région et chacune des organisations qui la composent. Les partenaires ont également convenu de travailler à un cadre de référence (ex. une charte, un code d'éthique ou autre) et de mécanismes pour aller chercher l'engagement de personnes et d'organisations à adhérer à ce cadre.

Le deuxième élément du scénario du futur vise à dégager des formes d'organisation du travail à promouvoir dans les entreprises : 
- Les partenaires s'engagent à développer des relations de travail axées sur le partenariat et la concertation en cherchant et en implantant des solutions procurant un bénéfice mutuel («gagnant/ gagnant ») à l'organisation et aux employés. Il est important de parvenir à une culture d'entreprise basée sur une démarche continue et concertée de résolution des problèmes entre les patrons et les syndiqués. Concrètement, cette concertation devra se réaliser par la mise en place de comités conjoints de gestion selon des zones de pouvoir à définir, de mécanismes de règlement de différends et de conflits basés sur des nouvelles approches de négociation ${ }^{4}$.

- Ils privilégient également des modes d'organisation du travail favorisant la mobilisation et la responsabilisation des employés ${ }^{5}$. Une organisation du travail mobilisatrice élaborée en concertation dans chacune des entreprises des secteurs publics et privés de notre région se traduira inévitablement par un enrichissement global des individus (les performances humaines) et un meilleur fonctionnement de ces entreprises (les performances productives). Les performances humaines et productives sont interdépendantes et évoluent en tandem

Le troisième élément du scénario du futur porte sur les infrastructures de soutien au travail. Sans en déterminer la constitution exacte, les partenaires ont identifié la nécessité de créer une sorte d'organisme de vigie qui aurait un rôle de "gardien des valeurs" auxquelles les partenaires ont adhéré. Cet organisme ferait également la promotion d'infrastructures sociales valorisant la mise en place d'un développement socio-économique axé sur la dimension humaine, c'est-à-dire tenant compte des valeurs humanistes des différents acteurs de ce développement.

Le quatrième élément du scénario du futur concerne l'emploi et la main-d'œuvre. Il mise sur la qualité du partenariat et de l'organisation du travail pour créer le plein emploi dans la région. Les partenaires s'engagent à continuer de valoriser des plans stratégiques de formation et de planification de la main-d'œuvre pour :

- l'accessibilité au marché du travail pour tous et toutes;
- le déploiement et l'adaptation de la main-d'œuvre selon les profils requis par les entreprises;

- les apprentissages par les futurs employés des aspects techniques et culturels du partenariat;

- le support des partenaires dans l'adhésion à une politique d'emploi axée particulièrement sur les jeunes;

- l'implication réelle et significative des institutions financières dans le développement régional.

\section{Il apparaissait stratégique d'élaborer avant tout une charte du travail énonçant les valeurs et l'éthique entre les partenaires du monde du travail.}

Dans les semaines suivant l'atelier, quatre groupes de travail ont été mandatés pour identifier et planifier les actions liées à la réalisation de chacun des consensus. Une équipe de coordination a assuré la rédaction du rapport d'atelier et la mobilisation des partenaires dans chacun des groupes de travail. Un consensus a été dégagé pour intensifier le travail du groupe de partenaires responsables du premier élément du scénario du futur. Il apparaissait stratégique d'élaborer avant tout une charte du travail énonçant les valeurs et l'éthique entre les partenaires du monde du travail. Le 25 juin 1998, plus de 35 partenaires se sont entendus sur cette charte du travail.

Au cours de cette réunion, il a été aussi décidé de réaliser une démarche de diffusion et d'adhésion de personnes et d'organisations à la déclaration d'engagement des partenaires du travail. Cette déclaration devait constituer un début d'implantation d'une culture de concertation devant guider et donner un sens aux actions quotidiennes des personnes et des entreprises de la région qui y adhèreront. Cette adhésion à une charte du travail rendra possible la diffusion, sur les plans régional, national et international, que les personnes et les entreprises de la région du SaguenayLac Saint-Jean valorisent une culture de concertation. Par conséquent, il sera plus évident d'investir dans une région ayant pour levier la concertation. 


\section{« DÉCLARATION D'ENGAGEMENT DES PARTENAIRES DU TRAVAIL DU SAGUENAY-LAC-SAINT-JEAN}

ATTENDU QUE le développement de notre région vise prioritairement le développement de l'emploi;

ATTENDU QUE le chômage et l'exclusion de personnes et de groupes de personnes ne favorisent pas la croissance économique et le développement social;

ATTENDU QUE nos discussions entre partenaires du travail ont soulevé de nombreuses inquiétudes au sujet de l'intégration des jeunes au marché du travail;

ATTENDU QUE des femmes et des hommes, partenaires du travail au Saguenay-Lac-Saint-Jean, voient la nécessité de se concerter relativement aux rapports qu'ils entretiennent entre eux et à ceux qu'ils voudraient développer au cours des prochaines années afin de soutenir le développement de notre région;

ATTENDU QUE la présente déclaration d'engagement est un guide ou une référence qui vise à créer un consensus, le plus large possible, autour d'objectifs et d'intérêts communs régionaux qui doivent se transposer dans la réalité et respecter les modes de fonctionnement de chaque organisation;

ATTENDU QUE toutes les personnes et les organisations qui ont un intérêt pour le travail sont invitées à adhérer, librement, à la présente déclaration d'engagement et qu'elles sont responsables de sa mise en æuvre par l'utilisation de moyens et de mécanismes appropriés.

\section{LES PARTENAIRES DU TRAVAIL DÉCLARENT CE QUI SUIT :}

\section{NOTRE RÉGION}

Nous, les partenaires du travail du Saguenay-Lac-Saint-Jean, misons sur le développement de notre région et voulons continuer à bâtir une société régionale équitable, sans exclusion, efficace et ouverte sur le monde.

Nous croyons que la concertation des partenaires du travail est un avantage stratégique majeur pour susciter l'investissement et soutenir le développement économique et social de notre région.

\section{NOTRE VISION}

Chaque personne est en droit d'aspirer au travail. La pauvreté et l'exclusion sont parmi les préoccupations majeures qui relèvent de notre responsabilité individuelle et collective.

La solidarité, la coopération et la concertation sont nécessaires à la mise en place d'une société régionale juste et équitable visant le plein emploi et la valorisation de nos ressources.

\section{NOS VALEURS}

Pour nous, le travail est une valeur fondamentale qui permet la valorisation des personnes et leur contribution au développement des organisations et de la société.

Nos relations de partenaires du travail sont fondées et se développent autour de l'intégrité, de la confiance, du respect mutuel, de la transparence et de la coopération entre les personnes et les organisations. Nos actions et décisions sont guidées par ces valeurs et témoignent de notre engagement.

\section{NOS MOYENS}

En privilégiant des communications ouvertes et transparentes, le développement des compétences et la participation des employés au processus décisionnel dans le cadre de leur travail, ainsi que des mécanismes de concertation dans nos organisations, nous favorisons l'amélioration de la qualité de vie au travail et l'atteinte de nos objectifs respectifs, tout en reconnaissant nos intérêts communs.

Nous reconnaissons l'importance d'instaurer et de développer des mécanismes et infrastructures qui assurent la promotion et le développement de nos valeurs et qui contribuent à la réalisation des engagements et objectifs auxquels nous avons souscrits et qui soutiennent notre vision.

\section{EN FOI DE QUOI, NOUS NOUS ENGAGEONS À METTRE EN GUVRE ET À PROMOUVOIR TOUS LES MOYENS APPROPRIÉS À LA RÉALISATION DES ENGAGEMENTS DES PARTENAIRES DU TRAVAIL. "}




\section{Conclusion}

La démarche prospective des partenaires du travail du Saguenay-Lac-Saint-Jean, amorcée en 1996, s'est arrêtée formellement en octobre 1998. Le collectif des partenaires du travail s'est dissous à la suite d'incidents critiques qui ont marqué les relations du travail dans des entreprises de la région. Cependant, les partenaires syndicaux et patronaux qui ont participé à l'élaboration du scénario du futur continuent de relever le défi de développer, par des actions précises, un avantage stratégique régional basé sur le potentiel humain en misant sur la qualité des interactions et de la concertation dans le monde du travail. Ils sont devenus des ambassadeurs d'une culture de concertation pour relever les défis de contrer l'exode de l'expertise des jeunes formés dans nos institutions d'enseignement, de rendre la région du Saguenay-LacSaint-Jean plus attrayante pour les investissements et pour la création d'entreprises. Cette démarche de mobilisation, basée sur une recherche constante d'une concertation entre les personnes dans les actions de développement, est devenue tout aussi importante que l'élaboration d'un plan d'action visant à réaliser les quatre éléments du scénario du futur 1998-2005.

Même si la démarche prospective semble avoir été oubliée dans sa forme anticipée, nous estimons que la pièce maîtresse de ce scénario du futur 1998-2005 que représente la déclaration d'engagement des partenaires du travail du Saguenay-Lac-saint-Jean est toujours actuelle... Chaque lecteur est convié à s'engager à actualiser et à promouvoir la « Déclaration d'engagement des partenaires du travail du SaguenayLac-Saint-Jean » jusqu'en 2025...

\section{Cette démarche de mobilisation, basée sur une recherche constante d'une concertation entre les personnes dans les actions de développement, est devenue tout aussi importante que l'élaboration d'un plan d'action visant à réaliser les quatre éléments du scénario du futur 1998-2005.}

\section{Notes et références}

1 Pierre Deschênes est professeur de psychologie organisationnelle à l'Université du Québec à Chicoutimi. Il a été, de 1996 à 1998, coordonnateur de ce rendez-vous des partenaires syndicaux et patronaux de la région du SaguenayLac-Saint-Jean.

2 Weisborg, M. et S. Janoff (1995). Future Search. An action Guide to Finding Common Ground in Organizations and Communities, San Francisco, Berrett-Koehler.

3 Beaulieu, J.P. et E.J. Carrière (2000). Mobiliser l'organisation face à son avenir : la démarche prospective, Montréal, Gaëtan Morin éditeur. Ces auteurs ont été les consultants qui ont animé la préparation et le déroulement de l'atelier de recherche du futur. Les pages 88 à 103 de leur volume décrivent les ingrédients de cette démarche prospective des partenaires du travail du Saguenay-Lac-SaintJean.

4 Deschênes, Pierre et coll. (2000). La négociation en relations du travail: nouvelles approches, Québec, Presses de l'Université du Québec (réédition corrigée de 1998).

Deschênes, P. (1999). «L'implantation d'une stratégie de négociation concertée dans une organisation: un guide d'utilisation ", revue Interactions, vol. $3, \mathrm{n}^{\text {os }} 1$ et 2 , p. 147167.

5 Conseil consultatif du travail et de la main-d'œuvre (1997). Document de réflexion : une nouvelle organisation du travail, Gouvernement du Québec. 